\title{
INFLUÊNCIA DA ENERGIA DE SOLDAGEM NAS CARACTERÍSTICAS GEOMÉTRICAS E ECONÔMICAS DE UMA JUNTA SOLDADA COM ELETRODO TUBULAR AUTOPROTEGIDO
}

\author{
Brites L.V.S.S. (') (lucasbritesufsj@hotmail.com), Silva E.M. ( $\left.{ }^{2}\right)$ (eduardosilva@ufsj.edu.br), \\ Silva,.S.C.(3)achaves@ufsj.edu.br, Brito T.G. (4) (tgbrito@unifei.edu.br)
}

(1)Universidade Federal de São João del-Rei (UFSJ)- DEMEC- Praça Frei Orlando, 170, Centro, São João del Rei, MG, CEP:36307352 (2)Universidade Federal de São João del-Rei (UFSJ)- DEMEC- Praça Frei Orlando, 170, Centro, São João del Rei, MG, CEP:36307352 (3)Universidade Federal de São João del-Rei (UFSJ)- DEMEC- Praça Frei Orlando, 170, Centro, São João del Rei, MG, CEP:36307352

(4)Universidade Federal de Itajubá (UNIFEI- ITABIRA) - DEMEC- Rua Irmã Ivone Drumond, 200-Distrito Indus. II, CEP:35903087

RESUMO: A soldagem é o principal processo de união permanente de peças metálicas, permitindo a montagem com rapidez, segurança e economia de material, entretanto as propriedades mecânicas de juntas soldadas de aços, em geral dependem fundamentalmente da microestrutura resultante na zona fundida ou do metal de solda e das diferentes regiões da zona termicamente afetada (ZTA), as quais, por sua vez, dependerão da composição química dos metais de base e de adição e da variação do ciclo térmico a que se submete a junta durante a soldagem. Neste interim, entre as opções de execução de soldagens, o processo com arame tubular vem crescendo em utilização em função de algumas peculiaridades. Este processo possibilita a formação de cordão com alta qualidade e bom aspecto visual, podendo ser utilizado em todas as posições de soldagem através de ajustes adequados de seus parâmetros operacionais. Deste modo, durante os experimentos, variou-se a tensão, a corrente, a distância bico de contato peça e a velocidade de alimentação do arame. Os dados foram submetidos a análises estatísticas utilizando-se um software comercial MINITAB14. Portanto, esse trabalho tem como objetivo fazer um estudo da influência da energia de soldagem nas características geométricas e econômicas de uma solda com arame tubular autoprotegido.

PALAVRAS-CHAVE: Soldagem, Arame tubular autoprotegido, Correlação

\section{INTRODUÇÃO}

A soldagem é um dos processos mais importantes de união de metais utilizados nas industrias. Esse método de união, em conjunto com a brasagem, têm importantes aplicações desde a indústria microeletrônica até a fabricação de navios e outras estruturas de maior porte. Sendo esse processo utilizado na fabricação de estruturas simples ou até mesmo em componentes com elevado grau de responsabilidade, como nas indústrias químicas, petrolíferas e nucleares (Marques etal,2017).

Assim atualmente, existem muitos processos de soldagem e podem ser classificados por diferentes critérios. Dentre eles, destacam-se os processos com arame tubular com proteção gasosa e autoprotegido os quais, vem crescendo sua demanda a cada ano, devido a sua versatilidade e produtividade.

Portanto, o processo com eletrodo tubular é um processo de soldagem por fusão, cujo arco é estabelecido entre a peça e o eletrodo alimentado continuamente, sendo protegido pela ação de um gás externo (ou não) e pela decomposição do fluxo alojado no interior do arame. Este fluxo é composto por materiais inorgânicos e metálicos que possuem várias funções, dentre as quais destacam-se a melhoria das características do arco elétrico e da transferência do metal de solda, a proteção do banho de fusão e, em alguns casos, a adição de elementos de liga, além de atuar como formador de escória (WELDING HANDBOOK, 1991; MOTA et al, 1998; BRACARENSE, 2000; 


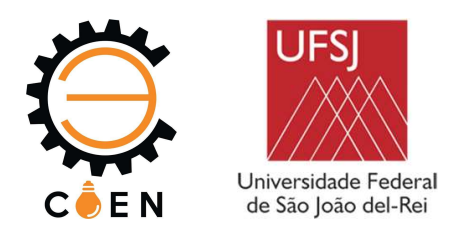

JOAQUIM, 2001; OLIVEIRA, 2002; FORTES 2004; STARLING etal,2005, STARLING etal, 2011, BRITES,2018).

Apesar da similaridade entre o processo eletrodo tubular com proteção gasosa e o processo com arame sólido- MIG/MAG, o processo com eletrodo tubular apresenta vantagens e limitações em relação ao arame sólido. Neste contexto, a sua resistividade elétrica é maior e, consequentemente, maior calor é gerado por efeito Joule como pode- se perceber pelas equações 1,2 e 3, podendo-se obter taxas de fusão 30 a 35\% superiores utilizando arames tubulares (Medeiros et al, 1989). Desta forma, os parâmetros de soldagem são extremamente importantes no sentido de melhor adequar o processo principalmente no que se refere aos modos de transferências (WANG, et al, 1995, SILVA, 2006) e seus reflexos na qualidade da solda.

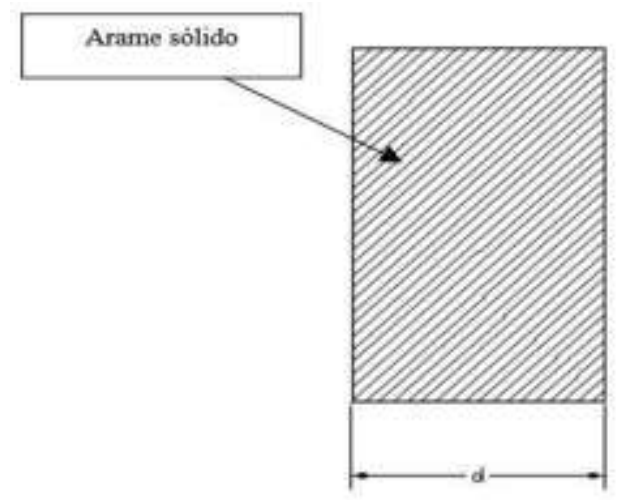

(a)

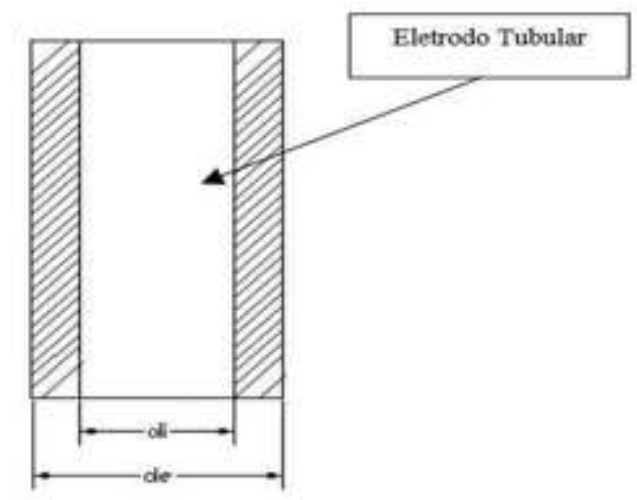

(b)

Figura 1- Comparação entre (a) o arame sólido do processo MIG/MAG com

(b) eletrodo tubular.

$$
Q_{T}=R_{e} I^{2} t
$$

Onde a Resistividade

Para o arame sólido

$$
R_{e}=\frac{\rho L}{A}
$$

$$
D e=\frac{\rho L}{\pi d^{2} / 4}
$$

Para o arame tubular,

$$
n=\frac{\rho L}{\pi_{e}\left(d-d_{1}{ }^{2}\right) / 4}
$$

Atualmente, o processo a arame tubular autoprotegido é aplicado principalmente na soldagem semi-automática, em substituição a soldagem manual com eletrodo revestido, onde são requeridas altas taxas de deposição e condições de tenacidade não muito severas, e na soldagem de grandes estruturas metálicas que exigem, muitas vezes, a sua realização em ambiente aberto sob forte ação do vento (OTEGUI, 1985; WIGERY, 1994). Na soldagem com arame tubular autoprotegido ometal fundido éprotegido porumacamadadeescória, pelos gases e pelos elementos desoxidantes/desnitretantes ( $\mathrm{Mn}, \mathrm{Al}, \mathrm{Ti}, \mathrm{Si}, \mathrm{Mg}$, etc.), que são resultantes da decomposição do fluxo. 


\section{REFERENCIAL TEÓRICO}

Segundo Montgomery (2012), a metodologia de Planejamento e Análise de Experimentos (DOE) é uma das principais estratégias disponíveis para a melhoria de processos. A partir, de uma análise sistematizada acerca de um problema, busca-se avaliar a magnitude de várias fontes de variação que influenciam um processo. Portanto, deve iniciar- se com a identificação e seleção dos fatores que possam contribuir para a variação, proceder- se, com à seleção de um modelo que inclua os fatores escolhidos e planejar experimentos eficientes para estimar seus efeitos. Assim, qualquer problema experimental deve ser sustentado por dois elementos: o projeto dos experimentos e a análise estatística dos dados. O emprego da abordagem estatística no Projeto e Análise de Experimentos necessita que as pessoas envolvidas nos experimentos tenham uma ideia bem clara a respeito do fenômeno que se pretende estudar, de como os dados serão coletados e de um entendimento básico das ferramentas de análise utilizadas. Conduzir adequadamente os experimentos, de acordo com o planejamento elaborado, garante o sucesso do problema em estudo ou, no mínimo, evita os riscos de insucesso da experimentação não planejada (Paiva, 2010).

Com relação aos projetos experimentais, tem-se que as técnicas mais utilizadas compreendem o planejamento Fatorial Completo, o Planejamento Fatorial Fracionado, os arranjos de Taguchi e a Metodologia de Superfície de Resposta. O arranjo mais comum é o fatorial completo, para o qual o número de experimentos é igual ao número de níveis experimentais, elevado ao número de fatores. No caso típico de fatoriais em dois níveis, o número de experimentos é dado por $\mathrm{N}=2 \mathrm{k}$.

Nesse sentido, nota-se que fatoriais completos cobrem todo o espaço experimental, entretanto, devido ao seu crescimento exponencial, arranjos com grande número de fatores podem tornar um processo de experimentação inviável. Ao passo que o planejamento fatorial fracionário segundo Montgomerye Runger (2003), se houver pouco interesse nas interações, essas podem ser negligenciadas, gerando-se frações do experimento completo sem comprometer, entretanto, a detecção da presença de fatores influentes, assim o número de experimentos é dado por N=2k-1. Entretanto, percebe-se que os pontos de ótimos geralmente são obtidos em superfícies em geral curvilíneas, côncavas, convexas, mistas ou em funções não lineares. Para tanto, uma evolução natural da prática experimental é migrar dos arranjos fatoriais para a Metodologia de Superfície de Resposta (MSR).

\subsection{Analise De Correlação}

A correlação entre as variáveis está presente em um grande número de processos de manufaturas, os quais apresentam respostas correlacionadas, como no caso da soldagem. Assim, a não observância desses relacionamentos entre as variáveis pode conduzir no processo a ótimos inapropriados, resultando em conclusões equivocadas e sem sentido, conforme observado por Paiva (2008), Wu (2005) e Khuri \& Cornell (1996). Deste modo, para correlacionar as variáveis foram utilizadas neste trabalho o coeficiente de correlação de Pearson "r de Pearson", o qual mede o grau da correlação e a direção dessa correlação - se positiva ou negativa entre duas variáveis. Este coeficiente assume apenas valores entre -1 e 1 Montgomery(2012).

$r=1$, significa uma correlação perfeita positiva entre as duas variáveis.

$r=-1$, significa uma correlação negativa perfeita entre as duas variáveis. Isto é, se uma aumenta a outra sempre diminui. 


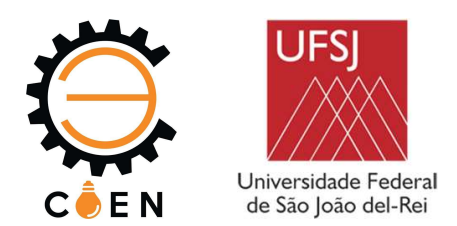

$\mathrm{r}=0$ significa que as duas variáveis não dependem linearmente uma da outra. No entanto, pode existir uma dependência não linear. Assim, o resultado $r=0$ deve ser investigado por outros meios.

O coeficiente de correlação de Pearson pode ser calculado pela Equação (4).

$$
r=\frac{\sum_{i=1}^{n}\left(x_{i}-\bar{x}\right)\left(y_{i}-\bar{y}\right)}{\sqrt{\sum_{i=1}^{n}\left(x_{i}-\bar{x}\right)^{2} \sum_{i=1}^{n}\left(y_{i}-\bar{y}\right)^{2}}}
$$

Onde $\mathrm{x} 1, \mathrm{x} 2, \ldots$, $\mathrm{xn}$ e $\mathrm{y} 1, \mathrm{y} 2, \ldots ; \mathrm{yn}$ são os valores medidos de ambas as variáveis. Assim temos a Eq. (5) e (6);

$$
\begin{aligned}
& \bar{x}=\frac{1}{n} \sum_{i=1}^{n} x_{1} \\
& \bar{y}=\frac{1}{n} \sum_{i=1}^{n} y_{1}
\end{aligned}
$$

Onde $\bar{x}, \bar{y}$ são oas médias aritméticas de ambas as variáveis.

O sinal de $\mathrm{r}$ indica a direção se a correlação é positiva ou negativa, o tamanho da variável indica a força da correlação. Portanto para interpretar a correlação de Pearson tem:

$r \geq 0,70$ para mais ou para menos indica uma forte correlação.

$0,30 \leq \mathrm{r} \leq 0,70$ positivo ou negativo indica correlação moderada.

$0 \leq \mathrm{r} \leq 0,30$ fraca correlação.

\subsection{Características geométricas}

a) Área total adicionada

$$
S_{i}=(S r+S p) m m^{2}
$$

Onde;

$\mathrm{Sr}$ - área de reforço $\left(\mathrm{mm}_{2}\right)$;

Sp- área de penetração $\left(\mathrm{mm}_{2}\right)$.

b) Índice de convexidade

$$
I C=\frac{r}{W} \times 100 \%
$$

Onde: $\mathrm{r}=$ reforço do cordão $(\mathrm{mm}) \mathrm{e} \mathrm{W}=$ largura do cordão $(\mathrm{mm})$.

\subsection{Características econômicas}

a) Taxa de deposição

$$
T D=3,6 \times \frac{\left(m_{f}-m_{i}\right)}{t}(\mathrm{~kg} / \mathrm{h})
$$

Onde:

IX COEN - Congresso de Engenharias da UFSJ Interconexão. 


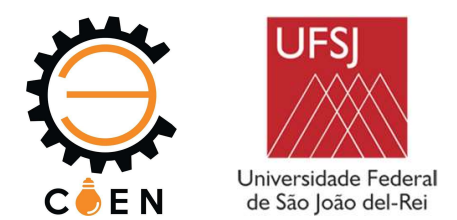

$\mathrm{mf}$ - massa do corpo de solda após soldagem $(\mathrm{g})$;

mi - massa dos corpos de prova antes da soldagem (g);

$\mathrm{t}$ - tempo de soldagem (s).

\section{b) Taxa de Fusão}

$$
T F=\frac{3,6 \times \rho a \times L}{t}(\mathrm{~kg} / \mathrm{h})
$$

Onde:

$\rho \mathrm{a}$ - densidade linear do arame $(3,3 \mathrm{~g} / \mathrm{m})$;

$\mathrm{L}$ - Comprimento do arame (mm).

\section{c) Rendimento}

$$
R=\frac{T D}{T F} \times 100 \%
$$

Onde:

TD- taxa de de posição(kg/h);

$\mathrm{TF}$ - taxa de fusão $(\mathrm{kg} / \mathrm{h})$.

\section{MATERIAIS E MÉTODOS}

Todas as sequências de testes foram realizadas utilizando o banco de ensaios do Laboratório de Soldagem da UFSJ - Campus Santo Antônio que foi desenvolvido no decorrer da pesquisa, o detalhamento é mostrado na Figura 2. A fonte de soldagem utilizada foi uma fonte MIG/MAG(MEGAPLUS250) com comando manual e funcionamento ajustado conforme o tipo de transferência a ser utilizado. A tocha de soldagem que guia o eletrodo consumível, conduza corrente elétrica e o gás de proteção para a área de trabalho, foi acoplada a um dispositivo de controle de velocidade de movimentação na posição plana, onde será possível ajustar a velocidade de soldagem nos níveis desejados através da utilização de um comando manual. De frente a tocha foi adaptada a um sistema para fixação dos corpos de prova. O comprimento do arame utilizado em cada teste foi definido pela velocidade de alimentação da máquina. O tempo de soldagem foi medido através de um cronômetro digital. Como material base utilizou-se chapas de aço ABNT-1020 com dimensões de 150x10x4 mm e $4 \mathrm{~mm}$ de espessura, esse aço tem baixa temperabilidade, resistência mecânica e tenacidade. Em seguida, estas amostras foram pesadas em uma balança de precisão e identificadas. Após, a identificação os corpos de prova, estes foram soldados com arame tubular autoprotegido. 

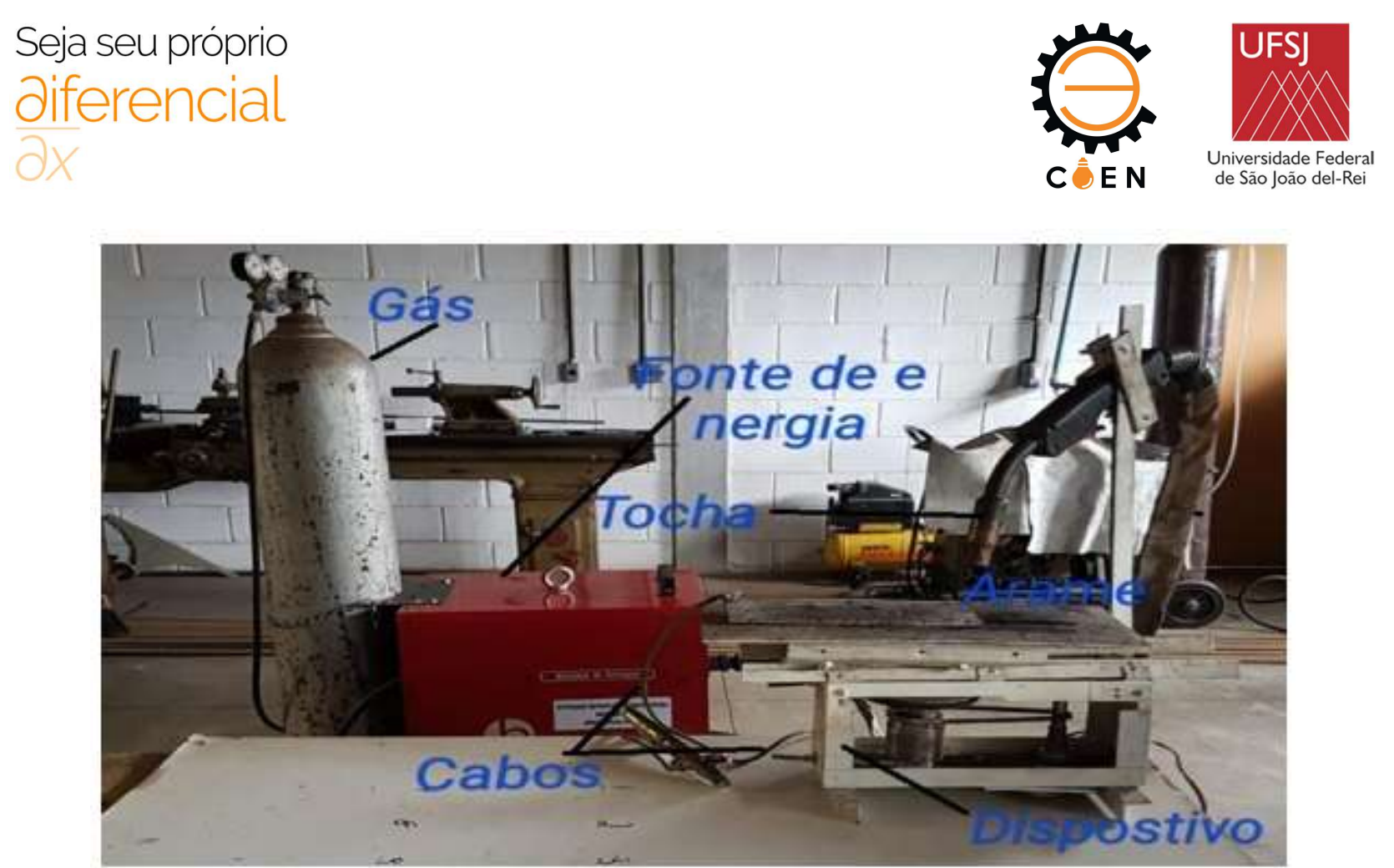

Figura 2. BancodeensaiosesquemáticoaserdesenvolvidonoLaboratóriodesoldagem-UFSJ.

Fonte: (Autor2019)

Tabela 1. Composição química do aço ABNT-1020- Fonte Villares

\begin{tabular}{|c|c|c|c|c|c|}
\hline \multicolumn{5}{|c|}{ Composição Química (\%) } \\
\hline $\mathrm{C}$ & $\mathrm{Mn}$ & $\mathrm{Cr}$ & $\mathrm{Ni}$ & $\mathrm{Mo}$ & Outros \\
\hline 0,20 & 0,45 & - & - & - & - \\
\hline
\end{tabular}

Como metal de adição utilizou-se o arame Solda Mig Tubular do Tipo Auto Protegido, classificado como E71T-GS, o qual dispensa o uso de cilindros de gás externo, pois o mesmo já possuiu na sua composição, componentes que protegem a solda, ideal para a máquina de solda MIG. Além disso, apresenta um arco suave, fácil remoção de escória e baixo índice de respingo.

Inicialmente em função do número de fatores a serem analisados (03) optou-se por utilizar o Delineamento dos Experimentos-fatorial fracionário (2n-1), onde $n$ é o número de parâmetros. Foram utilizados dois pontos centrais e uma replicagem, perfazendo um total de 18 experimentos, porém não foi satisfatória a aplicação da técnica estatística, pois a máquina de solda não permitia ajustes dos comandos fora dos tabelados. Assim utilizou-se dos parâmetros pré-definidos pelo fabricante perfazendo 12 experimentos. A partir do software comercial Minitab versão 14, foram processadas todas as análises estatísticas dos ensaios. Vale ressaltar que a fixação destes níveis foi possível somente após a realização de uma série de testes preliminares que permitiam obter, nas condições extremas de baixa energia e alta energia, o não escorrimento da poça de fusão.

Tabela 2. Composição química do metal de adição- Fonte Vonder

\begin{tabular}{|c|c|c|c|c|c|c|c|}
\hline \multicolumn{7}{|c|}{ Composição Química (\%) } \\
\hline $\mathrm{Fe}$ & $\mathrm{C}$ & $\mathrm{Mn}$ & $\mathrm{Si}$ & $\mathrm{S}$ & $\mathrm{P}$ & $\mathrm{Al}$ \\
\hline 98 & 0,13 & 0,85 & 0,45 & 0,013 & 0,016 & 1,23 \\
\hline
\end{tabular}




\begin{tabular}{|c|c|c|}
\hline \multicolumn{3}{|c|}{ Propriedades Mecânicas } \\
\hline Limite de escoamento (MPA) & Limite de ruptura (MPA) & Alongamento (\%) \\
\hline 400 & 480 & 20 \\
\hline
\end{tabular}

A Tabela 3 apresenta a matriz de experimentação, a qual mostra os fatores estudados bem como as respostas analisadas. Para determinar as características econômicas dos ensaios, inicialmente todos os corpos de proa foram pesados antes da soldagem. Após a soldagem houve a remoção da escória e respingos e foram pesados novamente. Em seguida foi avaliada aqualidade visual do cordão de solda, atribuindo notas de 0 a 10, considerando para isso critérios relativos à aparência do cordão, regularidade e formato do cordão, logo após os corpos de prova foram seccionados, lixados e polidos e atacados comnital $4 \%$.

Posteriormente através de um projetor de perfil com aumento de $20 \mathrm{X}$, foram fotografados (digitalizados) e transferidos para o Autocad (versão2015). Através da utilização das funções adequadas foi possível calcular a área total adicionada do cordão de solda (St) bem como as penetrações geradas conforme mostra o esquema da Figura 2. A partir destes dados determinaram-se as características econômicas do cordão conforme equações abaixo.

Tabela 3. Matriz de experimentação e resultados obtidos.

\begin{tabular}{|c|c|c|c|c|c|c|c|c|c|c|c|}
\hline \multicolumn{5}{|c|}{ Fatores } & \multicolumn{7}{|c|}{ Respostas } \\
\hline $\mathbf{T}$ & $\begin{array}{c}\mathrm{I} \\
\text { (A) }\end{array}$ & $\begin{array}{c}\mathrm{V} \\
\text { (Volts) }\end{array}$ & $\begin{array}{r}\text { DBCP } \\
(\mathrm{mm})\end{array}$ & $\begin{array}{c}\mathrm{Va} \\
(\mathrm{m} / \mathrm{min})\end{array}$ & $\begin{array}{l}\mathrm{Pa} \\
(\mathrm{g})\end{array}$ & $\begin{array}{l}\mathrm{Pd} \\
(\mathrm{g})\end{array}$ & Ts (s) & $\begin{array}{c}\mathrm{St} \\
(\mathrm{mm} 2)\end{array}$ & $\begin{array}{c}\mathrm{TD} \\
(\mathrm{Kg} / \mathrm{h})\end{array}$ & $\begin{array}{c}\mathrm{TF} \\
(\mathrm{Kg} / \mathrm{h})\end{array}$ & $\mathrm{R}(\%)$ \\
\hline 25 & 95 & 19 & 3 & 5,5 & 224,70 & 229,20 & 19,02 & 5,15 & 0,85 & 1,81 & 47,00 \\
\hline 26 & 95 & 19 & 3 & 6 & 182,9 & 191,57 & 19,53 & 8,50 & 1,59 & 2,23 & 71,52 \\
\hline 27 & 120 & 20 & 3 & 5,5 & 230,0 & 234,20 & 19,02 & 5,41 & 0,79 & 1,81 & 43,87 \\
\hline 28 & 120 & 20 & 3 & 6 & 184,80 & 190,51 & 19,00 & 10,70 & 1,08 & 2,23 & 48,43 \\
\hline 29 & 130 & 22 & 9 & 5,5 & 224,30 & 228,90 & 18,73 & 7,16 & 0,88 & 1,81 & 48,79 \\
\hline 30 & 130 & 22 & 9 & 6 & 184,20 & 192,54 & 20,26 & 9,53 & 1,48 & 2,23 & 66,33 \\
\hline 31 & 140 & 23 & 9 & 5,5 & 220,80 & 225,00 & 19,61 & 4,39 & 0,77 & 1,81 & 42,55 \\
\hline 32 & 140 & 23 & 9 & 6 & 183,70 & 190,07 & 19,33 & 9,93 & 1,18 & 2,23 & 53,10 \\
\hline 33 & 160 & 24 & 12 & 5,5 & 213,90 & 219,60 & 19,66 & 6,72 & 1,04 & 1,81 & 57,60 \\
\hline 34 & 160 & 24 & 12 & 6 & 182,40 & 190,09 & 19,76 & 6,48 & 1,40 & 2,23 & 62,74 \\
\hline 35 & 176 & 25 & 12 & 5,5 & 226,10 & 231,60 & 18,96 & 8,46 & 1,04 & 1,81 & 57,63 \\
\hline 36 & 176 & 25 & 12 & 6 & 184,60 & 194,96 & 20,69 & 8,63 & 1,80 & 2,23 & 80,68 \\
\hline
\end{tabular}

IX COEN - Congresso de Engenharias da UFSJ Interconexão. 


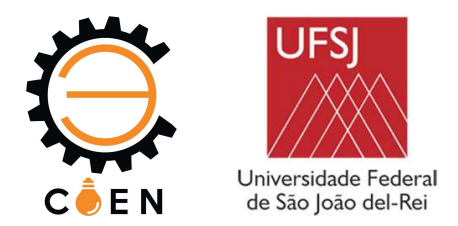

\section{RESULTADOS EDISCUSSÕES}

As respostas foram submetidas a análise de variância, de modo que pudesse avaliar os efeitos dos fatores de controle sobre as respostas, assim foi adotado um fator de confiabilidade de $95 \%$, adotando como nível de significância a 5\% utilizando o software Minitab 2014. A tabela 4 apresenta os resultados dessa análise, observa-se que os modelos desenvolvidos são adequados, pois o P-values é inferior a $5 \%$ e nota-se também que o R2(adj) está acima de $85 \%$, ou seja, o modelo desenvolvido tem um bom ajuste.

Tabela 4- Análise de Variancia para as respostas $\mathrm{IC}(\%) ; \mathrm{St}(\mathrm{mm} 2) ; \mathrm{TD}(\mathrm{kg} / \mathrm{h}) ; \mathrm{TF}(\mathrm{kg} / \mathrm{h}) ; \mathrm{E}(\mathrm{J} / \mathrm{mm}) ; \mathrm{R}$ $(\%)$

\begin{tabular}{|l|l|l|l|l|l|}
\hline Força & DF & SS & MS & F & P \\
\hline Fator & 6 & 35263,5 & 5877,3 & 79,47 & 0,000 \\
\hline Erro & 77 & 5684,6 & 74 & & \\
\hline Total & 83 & 40598,5 & & & \\
\hline $\mathrm{R}^{2}($ adj $)$ & & & & & 85,01 \\
\hline
\end{tabular}

Segundo Paiva (2008), a quase totalidade das pesquisas em otimização que utilizam alguma metodologia experimental para múltiplas respostas trata as respostas de forma isolada na fase de construção dos modelos de regressão. Este processo pode ser ineficiente, especialmente se as respostas forem fortemente correlacionadas. Assim a existência de correlações entre as várias respostas de um conjunto exerce uma forte influência sobre as funções de transferência utilizadas para representar as características de qualidade. Como o modelo matemático é extremamente e importante para a determinação do ponto de ótimo, a negligência da estrutura de correlação pode conduzir a pontos de ótimo inapropriados, fruto de uma inadequação do método dos mínimos quadrados ordinários Khuri \& Conlon (1981); Bratchell (1989). A tabela 5 apresenta a matriz de correlação das respostas analisadas, assim percebe-se que existe uma forte correlação positiva entre a taxa de deposição (TD) e a taxa de fusão(TF) em torno de $81 \%$, observa-se também uma correlação moderada negativa entre a diluição (D) e o índice de convexidade (IC), existe também uma correlação moderada entre o rendimento (R) e a taxa de fusão(TF),para o rendimento (R)e a taxa de deposição (TD) existe uma forte correlação entre as variáveis, a energia de soldagem (E) e o índice de convexidade(IC) existe uma forte correlação negativa e significativa em torno de $75 \%$.

Tabela 5- Análise de Correlação entre as Respostas.

\begin{tabular}{|c|c|c|c|c|c|c|}
\hline & IC & St & TF & TD & D & R \\
\hline & 0,276 & & & & & \\
St & 0,384 & & & & & \\
\hline & 0,258 & 0,712 & & & & \\
TF & 0,417 & 0,009 & & & & \\
\hline & 0,011 & 0,553 & 0,815 & & & \\
TD & 0,972 & 0,062 & 0,001 & & & \\
\hline
\end{tabular}




\begin{tabular}{|c|c|c|c|c|c|c|}
\hline $\mathbf{D}$ & $-0,657$ & $-0,017$ & 0,055 & 0,105 & & \\
\hline & 0,020 & 0,959 & 0,866 & 0,745 & & \\
$\mathbf{R}$ & $-0,105$ & 0,446 & 0,630 & 0,962 & 0,138 & \\
$\mathbf{E}$ & 0,745 & 0,146 & 0,028 & 0,000 & 0,668 & \\
\hline & $-0,757$ & $-0,031$ & $-0,154$ & 0,215 & 0,391 & 0,368 \\
\hline
\end{tabular}

(p)- Valor de P(P-Value)

Obs.: Valores em negritos na tabela são significativos, pois são menores que 5\%.

\subsection{Análise da correlação entre o índice de convexidade e a energia}

Segundo Zeemann (2003), um parâmetro de grande importância é a energia de soldagem pois juntamente com as características geométricas da junta e o nível de pré-aquecimento, é determinante nos ciclos térmicos impostos ao material e portanto, nas possíveis transformações microestruturais e no comportamento da junta. Assim ao avaliar a Figura 3 a qualmostra a correlação negativa entre o índice de convexidade eaenergia de soldagem, nota-se que quanto menor a energia de soldagem maior o índice de convexidade, pois existe um aumento no reforço, na penetração e largura da solda ao passo que aumenta a energia.

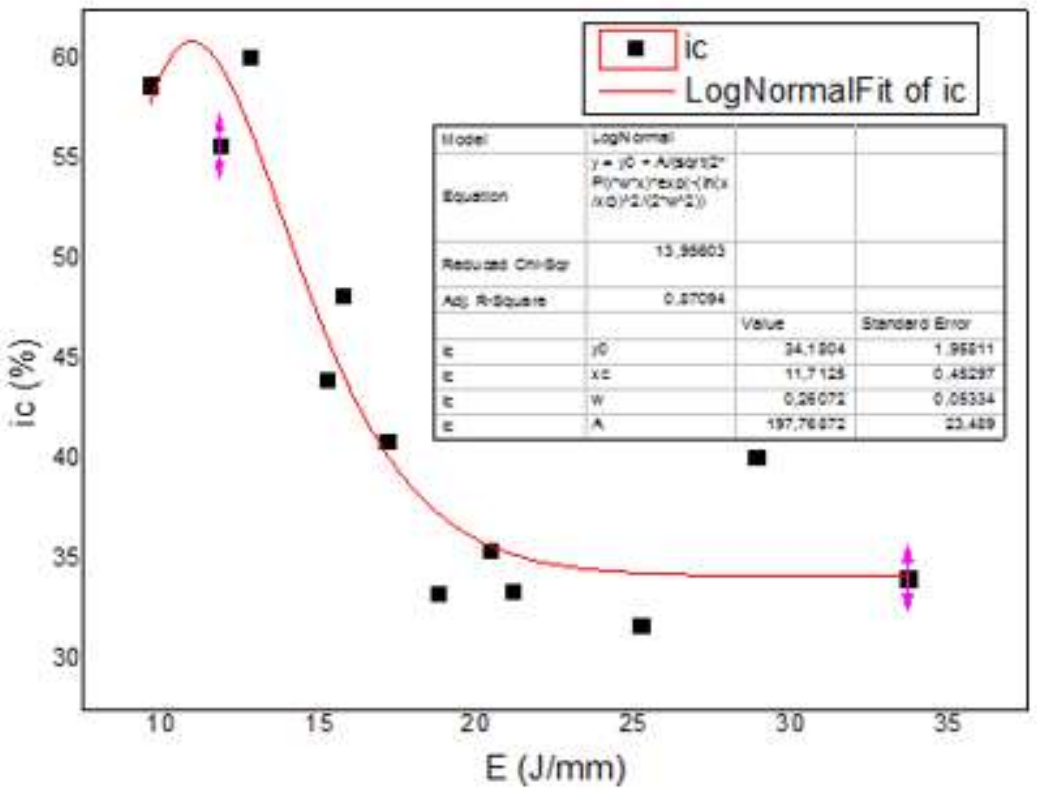

Figura 3. Correlação entre o índice de convexidade e a energia. Fonte: próprio autor

\subsection{Análise da correlação entre a diluição e a energia}

A diluição é um parâmetro que está ligado diretamente com a imposição de calor e com as características da poça de fusão, sendo a sua adequação importante na soldagem dos aços inoxidáveis 


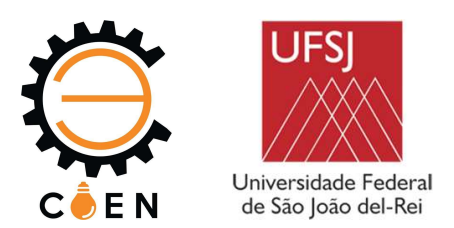

principalmente quando se utiliza o diagrama de Schaeffer, assim a composição química final da ZFdepende da intensidade destas interações, das composições químicas do metal de base, do metal de adição e também da participação relativa destes na formação da ZF. Portanto, o controle da diluição é importante na soldagem de metais dissimilares na deposição de revestimentos especiais sobre uma superfície metálica, na soldagem de metais de composição química desconhecida, caso muito comum em soldagem de manutenção e na soldagem de materiais que tenham teores de elementos prejudiciais à zona fundida como o carbono e o enxofre (MARQUES et al,2011). A figura apresenta o gráfico de contorno entre a energia de soldagem, o índice de convexidade do cordão e a diluição, observa-se que quanto maior a energia imposta maior a diluição e menor índice de convexidade do cordão. Percebe-se também que as maiores diluições ocorreram com a energia variando entre 20 a $25 \mathrm{~J} / \mathrm{mm}$.

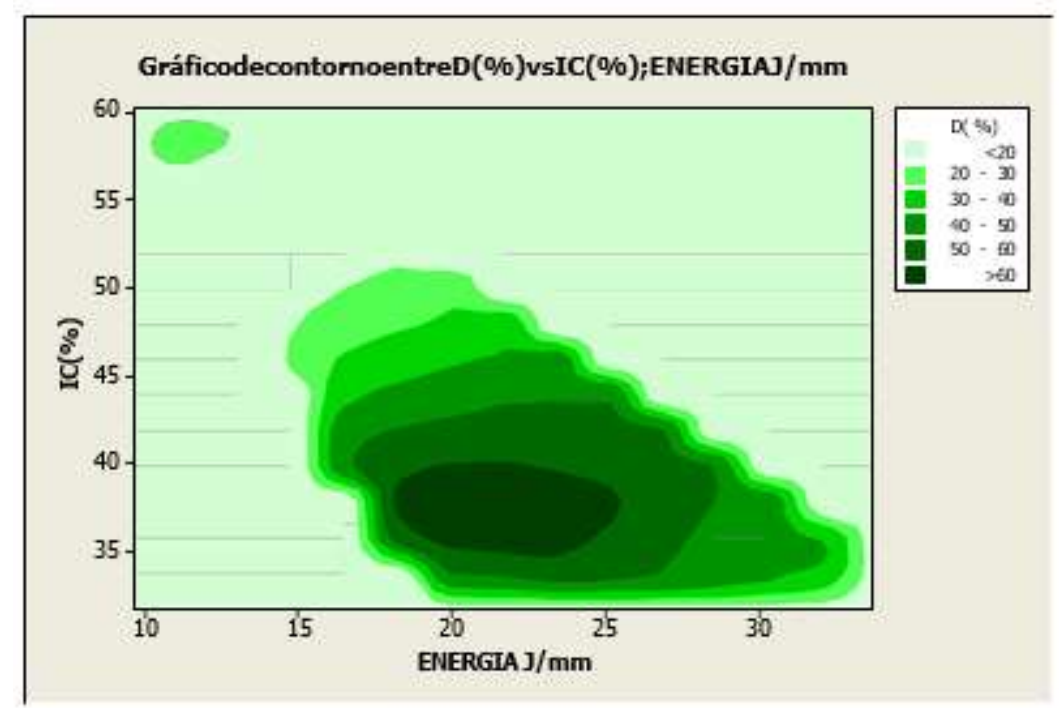

Figura 4. Gráfico de contorno ente a diluição versus o índice de convexidade e aenergia. Fonte: próprio autor

\subsection{Análise da correlação entre o rendimento e a energia}

O rendimento é uma das características importantes para avaliar o desempenho de um processo de soldagem, pois os mesmos influenciam diretamente na produtividade da soldagem. As perdas decorrentes da formação de respingos ou escórias são os fatores que, geralmente levam esses índices ao decréscimo Assim, a figura 5 apresenta uma relação entre o rendimento e a energia de soldagem, observa-se que ao aumentar a energia de soldagem existe maior imposição de calor e consequentemente maior o rendimento da soldagem, muito embora os maiores rendimentos alcançados com arame tubular autoprotegido giram em torno de $80 \%$ devido aos parâmetros de soldagem utilizados, pelas limitações da máquina de soldagem e também devido a escória gerada pelo processo, além do número de respingos. 


\section{Seja seu próprio
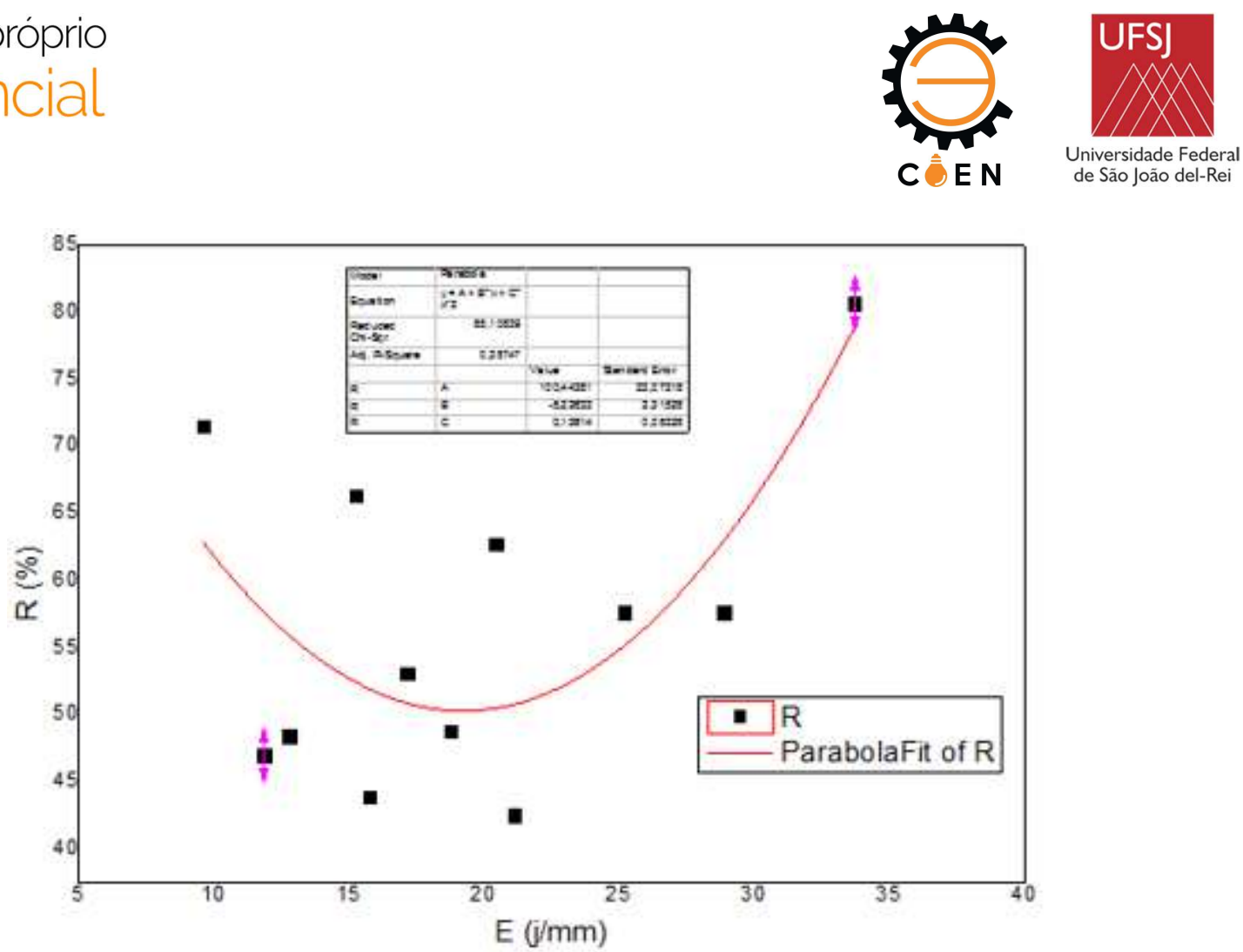

Figura 5. Gráfico de correlação entre o rendimento versus a energia de soldagem a.

Fonte: próprio autor.

\section{CONCLUSÕES}

Esse trabalho intitulado influência da energia de soldagem nas características geométricas e econômicas de uma junta soldada com eletrodo tubular autoprotegido nos permite concluir que:

Existe uma forte correlação positiva entre a taxa de deposição (TD) e a taxa de fusão (TF) em torno de $81 \%$, observa-se também uma correlação moderada negativa entre a diluição (D) e o índice de convexidade (IC), existindo também uma correlação moderada entre o uma forte correlação entre as variáveis, a energia de soldagem (E) e o índice de convexidade (IC) existe uma forte correlação negativa e significativa em torno de $75 \%$.

\section{DIREITOS AUTORAIS}

Os autores são os únicos responsáveis pelo conteúdo das informações contidas neste artigo.

\section{REFERÊNCIASBIBILOGRÁFICAS}

AMERICAN WELDING SOCIETY. Welding Process. In: AMERICAN WELDING SOCIETY. Welding Handbook, v.2, 8th ed., Miami: AWS, 1991. p.158-190.

BRATCHELL,N.,1989,“MultivariateResponseSurfaceModellingbyPrincipalComponents Analysis”, Journal of Chemometrics, Vol 3, pp.579-588

MARQUES, P.V.; MODENESI, P.J.; BRACARENSE, A.Q. Soldagem Mig/Mage com Arame Tubular. In: MARQUES, P.V.; MODENESI, P.J.; BRACARENSE,A.Q. Soldagem: Fundamentos $\quad \mathrm{e}$ Tecnologia. 1a. ed., Belo Horizonte: UFMG, 2005. p.233-261.

PAIVA, E.J. 2008., “Otimização de Processos de Manufatura com Múltiplas Respostas baseada em Índices de Capacidade", Dissertação de Mestrado, UNIFEI, 118p 


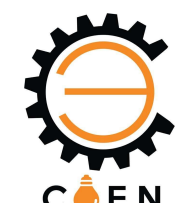

C E N

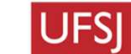

Universidade Federa de São João del-Rei

STARLING,C.M.D.; Modenesi, P.J.;BORBA,T.M.D.(2011),Cacterização do Cordão na SoldagemFCAWcomumArameTubular"MetalCored”,Soldag.insp.SãoPaulo,Vol.16,No.3, p.285-300,Jul/Set.

SILVA, E.M. ( 2006) , Comparação de Características Operacionais no Processo com Eletrodo Tubular utilizando as Transferências por Curto-Circuito e Pulsado na Posição Vertical Descendente. Dissertação de Mestrado, Universidade Federal de Itajubá, UNIFEI,109p.

Khuri,A.I.,Cornell,J.A.,1996, "Response Surfaces: Designs and Analyse”, Marcel Dekker Inc,2 Ed.,New York, USA,510p.

\section{INFLUENCE OF WELDING ENERGY ON GEOMETRIC AND ECONOMIC CHARACTERISTICS OF A WELDED JOINT WITH A SELF-PROTECTED TUBULAR ELECTRODE}

Brites L.V.S.S. (1) (lucasbritesufsj@hotmail.com), Silva E.M. (²) (eduardosilva@ufsj.edu.br), Silva,.S.C.(3)achaves@ufsj.edu.br, Brito T.G. (4) (tgbrito@unifei.edu.br)

(1)Universidade Federal de São João del-Rei (UFSJ)- DEMEC- Praça Frei Orlando, 170, Centro, São João del Rei, MG, CEP:36307352 (2)Universidade Federal de São João del-Rei (UFSJ)- DEMEC- Praça Frei Orlando, 170, Centro, São João del Rei, MG, CEP:36307352 (3)Universidade Federal de São João del-Rei (UFSJ)- DEMEC- Praça Frei Orlando, 170, Centro, São João del Rei, MG, CEP:36307352

(4)Universidade Federal de Itajubá (UNIFEI- ITABIRA) - DEMEC- Rua Irmã Ivone Drumond, 200-Distrito Indus. II, CEP:35903087

ABSTRACT: Welding is the main process of permanent joining of metal parts, allowing assembly with speed and safety and saving of material, however the mechanical properties of welded joints ofsteel in generaldepend fundamentally on the resulting microstructure in themelted zone or weld metaland in the different regions of the thermally affected zone (ZTA), which in turn will depend on the chemical composition of the base and addition metal sand the variation of the thermal cycle to which the joints subjected during welding. Thus, of welding, the process with tubular wire has been growing in use due to some peculiarities. This process allows the formation of a cord with high quality and good visual appearance, and can be used in all welding positions through appropriate adjustments of its operational parameters. Thus during the experiments, the voltage, current, distance part contact nozzle, and the feed speed of the wire. The data were submitted to statistical analysis using a MINITAB-14 commercial software. Therefore, this work a imps to study the influence of welding energy on the economic geometric characteristics of welding with self-protected tubular wire. Thus, there sults indicated that the best regulation of the welding parameters is essential for the optimization of the geometric characteristics of the cord and that there is a correlation between the welding energy and the economic characteristics of the cord.

KEYWORDS: Welding, self-Protected, Correlation. 